\section{(2) OPEN ACCESS}

\title{
Tau-induced focal neurotoxicity and network disruption related to apathy in Alzheimer's disease
}

\author{
Soichiro Kitamura, ${ }^{1,2}$ Hitoshi Shimada, ${ }^{1}$ Fumitoshi Niwa, ${ }^{3}$ Hironobu Endo, ${ }^{4}$ \\ Hitoshi Shinotoh, 1,5 Keisuke Takahata, ${ }^{1}$ Manabu Kubota, ${ }^{1}$ Yuhei Takado, ${ }^{1}$ \\ Shigeki Hirano, ${ }^{6}$ Yasuyuki Kimura, ${ }^{1,7}$ Ming-Rong Zhang, ${ }^{8}$ Satoshi Kuwabara, ${ }^{6}$ \\ Tetsuya Suhara, ${ }^{1}$ Makoto Higuchi ${ }^{1}$
}

\begin{abstract}
- Additional material is published online only. To view please visit the journal online (http://dx.doi.org/10.1136/ jnnp-2018-317970)
\end{abstract}

For numbered affiliations see end of article.

\section{Correspondence to}

Dr Hitoshi Shimada, Department of Functional Brain Imaging Research, National Institute of Radiological Sciences, National Institutes for Quantum and Radiological Science and Technology, Chiba 263-0024, Japan; shimada.hitoshi@qst. go.jp

Received 6 January 2018 Revised 16 April 2018 Accepted 15 May 2018 Published Online First 8 June 2018

Check for updates

To cite: Kitamura S, Shimada H, Niwa F, et al. J Neurol Neurosurg Psychiatry 2018:89:1208-1214.

\section{ABSTRACT}

Objective Apathy is a common neuropsychological symptom in Alzheimer's disease (AD), and previous studies demonstrated that neuronal loss and network disruption in some brain regions play pivotal roles in the pathogenesis of apathy. However, contributions of tau and amyloid- $\beta(A \beta)$ depositions, pathological hallmarks of $A D$, to the manifestation of apathy remain elusive. Methods Seventeen patients with AD underwent positron emission tomography (PET) with ${ }^{11} \mathrm{C}$-pyridinylbutadienyl-benzothiazole $3\left({ }^{11} \mathrm{C}-\mathrm{PBB} 3\right)$ and

${ }^{11} \mathrm{C}$-Pittsburgh compound-B ( ${ }^{11} \mathrm{C}$-PiB) to estimate tau and $A \beta$ accumulations using standardised uptake value ratio (SUVR) images. ${ }^{11} \mathrm{C}$-PBB3 and ${ }^{11} \mathrm{C}$-PiB SUVR were compared between $A D$ patients with high and low Apathy Scale (AS) scores. Additionally, volumetric and diffusion tensor MRI was performed in those areas where any significant difference was observed in PET analyses. Correlation and path analyses among AS and estimated imaging parameters were also conducted.

Results $A D$ patients with high $A S$ scores showed higher ${ }^{11} \mathrm{C}$-PBB3 SUVR in the orbitofrontal cortex (OFC) than those with low AS scores, while ${ }^{11} \mathrm{C}$-PiB SUVR in any brain regions did not differ between them. Elevated ${ }^{11} \mathrm{C}$-PBB3 SUVR in OFC, decreased OFC thickness and decreased fractional anisotropy (FA) in the uncinate fasciculus (UNC), which is structurally connected to OFC, correlated significantly with increased scores of the AS. Path analysis indicated that increased ${ }^{11} \mathrm{C}$-PBB3 SUVR in OFC affects apathy directly and through reduction of OFC thickness and subsequent decrease of FA in UNC.

Conclusions The present findings suggested that tau pathology in OFC may provoke focal neurotoxicity in OFC and the following disruption of the OFC-UNC network, leading to the emergence and progression of apathy in AD.

\section{INTRODUCTION}

Alzheimer's disease (AD), the most common cause of dementia, shows behavioural and psychological symptoms of dementia (BPSD) in addition to progressive cognitive dysfunction. Furthermore, BPSD among patients with mild cognitive impairment (MCI) are suggested to be a risk factor for conversion to AD. ${ }^{12}$ Partly due to a lack of understanding concerning the pathological basis of BPSD, some of the symptoms show limited response to drug treatment, resulting in the collapse of daily life or frequent hospitalisation. ${ }^{3}$ Among various appearances of BPSD, apathy is often seen in the course of AD. Apathy is defined as decreased motivation and lack of goal-directed behaviour, emotion or cognition. ${ }^{4} \mathrm{AD}$ patients with apathy show less interaction with family and other people as well as isolation from home and local societies; additionally, they are hardly able to ask for someone's help due to their lack of insight. Accordingly, apathy can be a cause of inadequate treatment and care $^{5}$ and also gives stress to caregivers. ${ }^{6}$

Some neuroimaging studies focusing on apathy in $\mathrm{AD}$ suggested that frontal dysfunction could be its neural basis. Previous studies revealed that apathetic $\mathrm{AD}$ patients showed decrement of grey matter volume, cerebral blood flow and glucose metabolism in some brain regions including the orbitofrontal cortex (OFC) and anterior cingulate cortex (ACC). ${ }^{7-9}$ Additionally, network disruption is assumed to be another key factor behind apathy in $\mathrm{AD}$. In diffusion tensor imaging (DTI) analyses, AD patients with apathy showed white matter abnormality in some tracts including the uncinate fasciculus (UNC) and anterior cingulate fasciculus. ${ }^{10}{ }^{11}$ Some of the above-mentioned brain regions and networks were mutually connected (ie, OFC and UNC) and seemed to play an important role in the appearance of apathy. However, associations among the pathological changes indicated above and the pathological cascade leading to apathy in $\mathrm{AD}$ still remain unclear.

Since depositions of amyloid- $\beta(\mathrm{A} \beta)$ and hyperphosphorylated tau concurrently occur in $\mathrm{AD}$ brain, ${ }^{12}$ they are assumed to have crucial importance in the development of BPSD, including apathy. We hypothesised that these abnormal proteins in the focal brain region would evoke network disruption subsequent to focal neural damage, leading to apathy. Recent advancements in positron emission tomography (PET) imaging technique visualising $\mathrm{A} \beta$ and tau have enabled us to explore the association between these abnormal proteins and clinical manifestations in vivo. We have also developed a tau PET ligand, ${ }^{11} \mathrm{C}$-pyridinyl-butadienyl-benzothiazole $3\left({ }^{11} \mathrm{C}\right.$-PBB3), that shows high affinity and selectivity for tau deposits ${ }^{13}{ }^{14}$ and reported that tau deposition measured by ${ }^{11} \mathrm{C}-\mathrm{PBB} 3$ showed close relation to cognition of $\mathrm{AD}$ and cognitively healthy elderly. ${ }^{15}$ While some previous studies investigated the association between $\mathrm{A} \beta$ deposition and apathy 
using amyloid PET, no study has assessed whether either or both $\mathrm{A} \beta$ and tau pathologies might have some influence on the damage to focal brain and connected fibres in $\mathrm{AD}$ with apathy.

To assess whether focal depositions of $A \beta$ and tau are associated with the emergence of apathy, we performed a PET scan with ${ }^{11} \mathrm{C}$-PBB3 and ${ }^{11} \mathrm{C}$-Pittsburgh compound-B $\left({ }^{11} \mathrm{C}\right.$-PiB $)$ in $\mathrm{AD}$ patients with high and low Apathy Scale (AS) scores. ${ }^{16}{ }^{17}$ We also investigated the association among focal $\mathrm{A} \beta$ and tau deposits, neural loss of focal brain, disruption of connected fibre and the severity of apathy.

\section{METHODS}

\section{Participants}

We recruited 11 clinically diagnosed $\mathrm{AD}$ dementia and $15 \mathrm{MCI}$ subjects from Chiba University Hospital and affiliated hospitals between March 2013 and March 2014. Diagnosis of AD was based on the National Institute of Neurological and Communicative Disease and Stroke/Alzheimer's Disease and Related Disorders Association criteria ${ }^{18}$ and that of MCI as meeting the Petersen's criteria. ${ }^{19}$ Since we intended to focus on AD spectrum patients, seven patients with no clear cortical ${ }^{11} \mathrm{C}-\mathrm{PiB}$ retention were excluded from the assay group. We also excluded two other patients because their severely impaired cognitive status hampered collections of some neuropsychological data. Thus, 17 of 26 patients with $\mathrm{AD}$ (8 $\mathrm{AD}$ dementia and $9 \mathrm{MCI}$ due to $\mathrm{AD}$ ) were enrolled for further analyses. Their general cognitive function and activity of daily living were evaluated by Mini-Mental State Examination (MMSE) ${ }^{20}$ and Clinical Dementia Rating scale. ${ }^{21}$ Psychological assessments for apathy and depression were performed using $\mathrm{AS}^{1617}$ and Geriatric Depression Scale (GDS), ${ }^{22}$ respectively. AS is composed of 14 items (8 positive and 6 negative symptoms evaluating motivation or interest). Subjects rate each questionnaire item at $0-3$, and the total score ranges from 0 to 42 . According to a previous report, patients with high AS scores were defined as having AS $\geq 16$ and the others as those with low AS scores..$^{23}$ Frontal lobe functions of the patients were also evaluated by frontal assessment battery (FAB). ${ }^{24}$

\section{Imaging procedure}

PET scans

${ }^{11} \mathrm{C}-\mathrm{PBB} 3$ and ${ }^{11} \mathrm{C}-\mathrm{PiB}$ were radiosynthesised by the Department of Radiopharmaceuticals Development at NIRS, following a previously reported protocol. ${ }^{132526}$ Seventy-minute dynamic PET scans were performed after an intravenous injection of ${ }^{11} \mathrm{C}$ PBB3 (injected dose: $436 \pm 55 \mathrm{MBq}$; molar activity: $82 \pm 33 \mathrm{GBq} /$ $\mu \mathrm{mol}$ ) or ${ }^{11} \mathrm{C}-\mathrm{PiB}$ (injected dose: $378 \pm 38 \mathrm{MBq}$; molar activity: $85 \pm 50 \mathrm{GBq} / \mu \mathrm{mol}) .{ }^{11} \mathrm{C}-\mathrm{PBB} 3$ was injected under dim lighting to avoid its photoracemisation. ${ }^{13}$ PET images were acquired by ECAT Exact HR + system (Siemens AG) with an axial field of view of $155 \times 155 \mathrm{~mm}$, providing 63 contiguous slices. The intrinsic spatial resolution was $4.3 \mathrm{~mm}$ in-plane and $4.2 \mathrm{~mm}$ fullwidth at half-maximum axially. Subjects' heads were restrained with a band extending across the forehead and attached to the headrest. Head movements were carefully monitored with laser beams during the PET scans, and corrections of head position were made in case of movement. PET images were reconstructed by the filtered back-projection method with corrections for attenuation and scatter.

\section{MRI acquisition}

3T MR images were acquired with Signa HDx (GE Healthcare, Wisconsin, USA). Subjects were scanned with a 3D T1-weighted gradient echo sequence (repetition time $(\mathrm{TR})=7 \mathrm{msec}$, echo time $(\mathrm{TE})=2.8 \mathrm{msec}$, field of view $(\mathrm{FOV})=260 \mathrm{~mm} \times 244 \mathrm{~mm}$, matrix $=256 \times 256,170$ contiguous axial slices of $1.0 \mathrm{~mm}$ thickness). Additionally, we performed DTI using an echo planar imaging sequence $(\mathrm{TR}=10000 \mathrm{msec}, \mathrm{TE}=85 \mathrm{msec}$, $\mathrm{b}=1000 \mathrm{~s} / \mathrm{mm}^{2}, 25$-axis encoding, FOV $=260 \mathrm{~mm} \times 260 \mathrm{~mm}$, matrix $=128 \times 128$, slice thickness $=3.0 \mathrm{~mm}, 36$ contiguous axial slices).

\section{Image analyses}

PET image analysis

A motion correction was applied for dynamic PET data, and standard uptake value ratio (SUVR) images were generated using cerebellar grey matter as a reference region. In order to create SUVR images, we applied voxel-based calculation of ${ }^{11} \mathrm{C}$-PBB3-PET data at $30-50 \mathrm{~min}$ and ${ }^{11} \mathrm{C}$-PiB-PET data at 50-70 min. Next, SUVR images were coregistered to the individual T1-weighted MR images, followed by anatomical normalisation of these images to Montreal Neurological Institute 152 standard space by a Diffeomorphic Anatomical Registration Through Exponentiated Li Algebra algorithm. ${ }^{27}$ Region of interests (ROIs) of the OFC and ACC, previously reported as crucial regions for emerging apathy, ${ }^{7-9}$ were defined according to the automated anatomical labelling atlas. ${ }^{28}$ Additionally, ROIs of frontal (including lateral, orbital, medial frontal and frontal-related gyri), medial temporal (including hippocampus, parahippocampus, amygdala and fusiform gyrus), lateral temporal (including superior, middle and inferior temporal gyri), parietal (including superior and inferior parietal gyri, supramarginal and angular gyri) and occipital (including superior, middle and inferior occipital gyri) cortices were defined in order to confirm tau and $A \beta$ accumulations in each region. These ROIs were intersected by individually normalised grey matter mask images and applied to normalised SUVR images for calculating the mean SUVR value. All images were processed using PMOD software V.3.7 (PMOD Technologies) and Statistical Parametric Mapping software (SPMV.12, Wellcome Trust Centre for Neuroimaging, UK).

\section{Cortical thickness measurement}

Cortical reconstruction and estimation of cortical thickness were performed using FreeSurfer software (V.5.3.0) (http://surfer.nmr. mgh.harvard.edu/). The detailed process of the automated surface reconstruction pipeline has been described in previous publications. ${ }^{29}{ }^{30}$ Briefly, T1-weighted MRI images were processed by non-brain tissue removal, automated Talairach transformation, segmentation of subcortical white matter and deep grey matter structures, tessellation of the gray-white matter boundary and surface deformation to optimally place the grey/white and grey/ cerebrospinal fluid borders. Then, cortical thickness was calculated as the closest distance from the grey/white boundary to the grey/cerebrospinal fluid (CSF) boundary at each vertex on the surface. The Desikan-Killiany cortical atlas implemented in FreeSurfer was used to evaluate the thickness in the target region where significant uptake of PET ligand was observed in $\mathrm{AD}$ patients with high AS scores relative to those with low AS scores. ${ }^{31}$

\section{DTI analysis}

A DTI analysis was performed using FSL software (www.fmrib. ox.ac.uk). In preprocessing, images were corrected for eddy current and head motion with FMRIB's Diffusion Toolbox (FDT), and brain tissue extraction was performed with the Brain Extraction Toolbox implemented in FSL. ${ }^{32}$ Using the DTIFIT 
Reconstruct tool in FDT, a diffusion tensor model was fitted at each voxel, and a fractional anisotropy (FA) map was generated. Probabilistic tractography was performed using FDT. We placed a seed ROI in a target region identified as an area associated with apathy in the above-mentioned PET analyses. A waypoint ROI was also defined in an area structurally connected with the target region. Probabilistic streamlines through each voxel by repetitive sampling from the principal direction were computed from the seed ROI through the waypoint ROI. This algorithm was performed with a tract curvature threshold of $0.2 \mathrm{~mm}$, maximum number of steps of 2000 , step length of $0.5 \mathrm{~mm}$ and 5000 samples, and generated connectivity streamlines were thresholded at a value equal to $5 \%$ of the maximum voxel intensity value in each subject in order to exclude spurious streamlines.

\section{Statistical analysis}

Group differences in demographic characteristics including age, education period, MMSE, FAB, AS and GDS were examined with unpaired two-tailed t test between $A D$ patients with high and low AS scores and with Fisher's exact test for gender. To examine group differences in the estimated mean ${ }^{11} \mathrm{C}-\mathrm{PBB} 3$ and ${ }^{11} \mathrm{C}-\mathrm{PiB}$ SUVR values of each volume of interest (VOI), unpaired two-tailed t-test was performed with Bonferroni correction to avoid type I errors due to multiplicity $(p \leq 0.0071$ as significant). We defined VOI(s) with significant uptake of PET ligand in $\mathrm{AD}$ patients with high AS scores relative to those with low AS scores as target region(s). We also tested group differences in the estimated mean cortical thickness of target region and mean FA values of the fibre connected with the target region by unpaired two-tailed t-test $(\mathrm{p}<0.05)$. Additionally, we analysed the relationship between AS and each estimated parameter using partial correlation analysis with age as covariate. In addition to the above-mentioned group-wise comparisons, correlations of AS scores with PET and MRI measures were analysed in all patients with $\mathrm{AD}$ examined here, since AS score is a variable continuously distributed among these cases and is accordingly a quantitative index representing the magnitude of apathy even if the value is below the cut-off ( $<16$ points). Moreover, a structural equation modelling path analysis was performed to assess the relationships among AS scores, mean ${ }^{11} \mathrm{C}$-PBB3 and ${ }^{11} \mathrm{C}-\mathrm{PiB}$ SUVR values in the target region, mean cortical thickness of the target region and mean FA values of the fibres connecting the target and waypoint regions. To validate the model fitting, we conducted $\chi^{2}$ test, comparative fit index (CFI) and root mean square error approximation (RMSEA). We calculated Akaike's information criterion (AIC) to determine a better model. A good fit of the model was defined by no significant results $(p>0.05)$ of the $\chi^{2}$ test, CFI $>0.97$, RMSEA $<0.05$ and a relatively small AIC. Statistical and path analyses were performed using SPSS V.22 and AMOS V.22, respectively.

\section{RESULTS}

\section{Demographic data}

Table 1 shows the demographic data in this study. There was no significant difference in age, education period, gender, MMSE, $\mathrm{FAB}$ and GDS between the two patient groups $(\mathrm{p} \geq 0.058)$. There was no significant association of AS with GDS $(d f=17, r=0.43$, $\mathrm{p}=0.087)$, MMSE $(d f=17, r=-0.22, \mathrm{p}=0.39)$ or FAB $(d f=17$, $r=-0.41, \mathrm{p}=0.102$ ) by Pearson's correlation analysis.

Demography of nine age-matched healthy controls without cognitive deficits, who were selected from participants in our previous study, ${ }^{15}$ is also displayed in table 1 . We used PET measures of these subjects as references to confirm accumulations of tau and $\mathrm{A} \beta$ deposits in the brains of currently recruited $\mathrm{AD}$ cases. However, thorough comparisons between $\mathrm{AD}$ and control subjects were not performed, as a significant subset of clinical and image data, including AS scores and DTI images, were not available in these controls.

\section{Group comparisons of estimated PET and MRI data}

Table 2 shows the estimated imaging parameters in AD patients with low and high AS scores, along with control data derived from our previous work. ${ }^{15}$ PBB3 SUVRs were significantly elevated in both $\mathrm{AD}$ groups with low and high AS scores compared with cognitively healthy controls. As documented elsewhere, ${ }^{15}$ PBB3 SUVR in the cerebral cortex of control subjects were smaller than 1.0, since non-displaceable radioligand retention in the cerebellum was greater than those of neocortical and limbic cortices. Likewise, PBB3 SUVRs in several target regions of AD patients with low AS scores were found to be below 1.0. Despite this observation, AD patients with low AS scores exhibited significantly higher PBB3 SUVRs in the parietal $(0.97 \pm 0.09$ vs $0.83 \pm 0.05)$, lateral temporal $(1.05 \pm 0.06$ vs $0.95 \pm 0.04)$ and occipital $(1.05 \pm 0.07$ vs $0.90 \pm 0.03)$ cortices in comparison with control subjects $(\mathrm{p}<0.0071$ by t-test with Bonferroni correction). AD patients with high AS scores showed even larger PBB3 SUVR values in the frontal cortex and OFC than patients with low AS scores $(p=0.006$ and $p=0.007$, respectively). None of the other regions showed significant differences in mean ${ }^{11} \mathrm{C}$ PBB3 SUVR values between the two patient groups $(p \geq 0.015)$.

Representative ${ }^{11}$ C-PBB3 SUVR images of the AD patients with low and high AS scores were shown in panels $1 \mathrm{~A}$ and $1 \mathrm{~B}$ of figure 1 , respectively. In line with ROI-based analyses,

Table 1 Clinical and psychological data of current subjects

\begin{tabular}{lcccccc} 
& $\begin{array}{l}\text { Total } \\
(\mathbf{n}=17)\end{array}$ & $\begin{array}{l}\text { High AS scores } \\
(\mathbf{n}=10)\end{array}$ & $\begin{array}{l}\text { Low AS scores } \\
(\mathbf{n}=7)\end{array}$ & $\boldsymbol{t}$ & P values & HC (n=9) \\
\hline Clinical stage: AD (MCI) & $8(9)$ & $6(4)$ & $2(5)$ & & - \\
\hline Age: mean (SD) & $70.7(8.4)$ & $68.3(10)$ & $74.1(2.7)$ & -1.67 & 0.123 & $66.5(6.2)$ \\
\hline Years of schooling: mean (SD) & $12.2(2.1)$ & $12.1(2.1)$ & $12.4(2.2)$ & -0.31 & 0.760 & $14.7(2.0)$ \\
\hline Sex: male (\%) & $7(41)$ & $2(20)$ & $5(71)$ & & 0.058 & $7(78)$ \\
MMSE: mean (SD) & $21.4(6.5)$ & $19.7(6.0)$ & $23.7(6.9)$ & -1.29 & 0.218 & $29.4(0.7)$ \\
FAB: mean (SD) & $12.8(3.5)$ & $11.7(3.9)$ & $14.3(2.2)$ & -1.74 & 0.103 & - \\
\hline AS: mean (SD) & $14.1(6.2)$ & $18.3(1.6)$ & $8.0(5.0)$ & 5.24 & $0.001^{*}$ & - \\
\hline GDS: mean (SD) & $3.8(2.7)$ & $4.5(2.4)$ & $2.7(2.8)$ & 1.40 & 0.181 & - \\
\hline
\end{tabular}

$A D$, Alzheimer's disease; AS, Apathy Scale; FAB, frontal assessment battery; GDS, Geriatric Depression Scale; $\mathrm{HC}$, cognitively healthy control; MCl, mild cognitive impairment; MMSE, Mini Mental State Examination. 
Table 2 Group comparison of estimated PET and MRI data between AD patients with high and low AS scores

\begin{tabular}{|c|c|c|c|c|c|}
\hline & \multicolumn{2}{|l|}{ Patients with AD } & \multirow[b]{2}{*}{$t$} & \multirow[b]{2}{*}{$P$ values } & \multirow[b]{2}{*}{ HC } \\
\hline & High AS scores & Low AS scores & & & \\
\hline \multicolumn{6}{|l|}{${ }^{11}$ C-PBB3 SUVR } \\
\hline Frontal cortex & $1.10(0.11)$ & $0.94(0.09)$ & 3.16 & $0.006^{*}$ & $0.86(0.04) \dagger$ \\
\hline Lateral temporal cortex & $1.17(0.12)$ & $1.05(0.06)$ & 2.77 & 0.015 & $0.95(0.04) \dagger \ddagger$ \\
\hline Medial temporal cortex & $1.09(0.10)$ & $1.02(0.05)$ & 1.56 & 0.140 & $0.99(0.05) \dagger$ \\
\hline Parietal cortex & $1.08(0.10)$ & $0.97(0.09)$ & 2.16 & 0.048 & $0.83(0.05) \dagger \ddagger$ \\
\hline Occipital cortex & $1.14(0.11)$ & $1.05(0.07)$ & 1.96 & 0.069 & $0.90(0.03) \dagger \ddagger$ \\
\hline Orbitofrontal cortex & $1.14(0.98)$ & $0.98(0.07)$ & 3.12 & $0.007^{*}$ & $0.92(0.05) \dagger$ \\
\hline Anterior cingulate cortex & $1.12(0.17)$ & $0.94(0.10)$ & 2.45 & 0.027 & $0.91(0.07) \dagger$ \\
\hline \multicolumn{6}{|l|}{${ }^{11} \mathrm{C}$-PiB SUVR } \\
\hline Frontal cortex & $1.96(0.43)$ & $1.79(0.47)$ & 0.80 & 0.438 & $1.00(0.05) \dagger \ddagger$ \\
\hline Lateral temporal cortex & $1.96(0.46)$ & $1.86(0.42)$ & 0.46 & 0.656 & $1.02(0.04) \dagger \ddagger$ \\
\hline Medial temporal cortex & $1.56(0.26)$ & $1.50(0.23)$ & 0.52 & 0.611 & $1.07(0.04) \dagger \ddagger$ \\
\hline Parietal cortex & $1.93(0.40)$ & $1.85(0.49)$ & 0.34 & 0.736 & $0.95(0.05) \dagger \ddagger$ \\
\hline Occipital cortex & $1.74(0.31)$ & $1.65(0.27)$ & 0.62 & 0.542 & $0.97(0.05) \dagger \ddagger$ \\
\hline Orbitofrontal cortex & $2.01(0.45)$ & $1.81(0.46)$ & 0.87 & 0.397 & $1.01(0.11) \dagger \ddagger$ \\
\hline Anterior cingulate cortex & $2.31(0.57)$ & $2.05(0.56)$ & 0.91 & 0.377 & $1.17(0.07) \dagger \ddagger$ \\
\hline
\end{tabular}

Values are listed as mean (SD).

*Low AS scores<highAS scores.

$+\mathrm{HC}<$ high AS scores.

$\neq H C<$ lowAS scores $(p<0.071)$.

${ }^{11} \mathrm{C}-\mathrm{PBB} 3,{ }^{11} \mathrm{C}$-pyridinyl-butadienyl-benzothiazole $3 ;{ }^{11} \mathrm{C}-\mathrm{PiB},{ }^{11} \mathrm{C}-$ Pittsburgh compound-B; AD, Alzheimer's disease; AS, Apathy Scale; HC, cognitive healthy control; PET, positron emission tomography; SUVR, standard uptake value ratio.

voxel-by-voxel comparisons of PBB3 SUVR images between the two patient groups by statistical parametric mapping revealed that AD patients with high AS scores presented significantly greater radioligand retention in OFC than patients with low AS scores (figure $1 \mathrm{C}$ ). In the ${ }^{11} \mathrm{C}-\mathrm{PiB}$ SUVR analyses, no cortical regions showed differences between the two groups $(p \geq 0.397)$.

Since OFC was extracted as a target region in the PET study, the OFC label was defined as adding lateral and medial division of bilateral OFC, which was applied to the measurement of mean cortical thickness in each participant. Furthermore, we focused on UNC because this area is associated with apathy in patients with $\mathrm{AD}$ and is structurally connected to OFC. ${ }^{33}$ To perform DTI analysis in UNC, we set a seed ROI in white matter on a coronal plane at the tip of the inferior horn of the lateral ventricle and a waypoint ROI in white matter on a coronal plane at the tip of the frontal horn of the lateral ventricle. ${ }^{34}$ The left and right UNCs were generated independently and were then added and binarised as bilateral UNC masks. We applied these masks to the original FA map and calculated the mean FA value of UNC. The mean OFC thickness and mean FA value of UNC did not significantly differ between $\mathrm{AD}$ patients with high and low AS scores $(p=0.068$ and $p=0.054$, respectively).

\section{Analyses of correlations between AS and neuroimaging} indices

Partial correlation analysis revealed a significant positive correlation between AS scores and mean ${ }^{11}$ C-PBB3 SUVR in OFC (figure 2A). Mean OFC thickness and mean FA values of UNC showed a significant negative correlation with AS scores (figure 2B,C). There was no significant correlation between AS scores and mean ${ }^{11} \mathrm{C}-\mathrm{PiB}$ SUVR values in OFC $(r=0.22$, $\mathrm{p}=0.408)$.
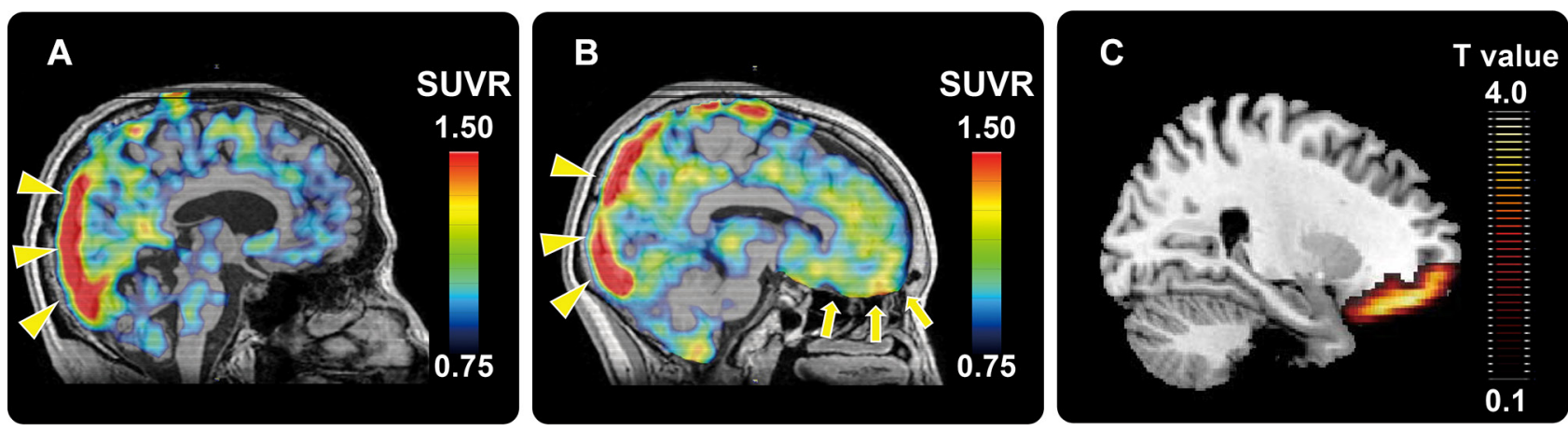

Figure 1 Representative ${ }^{11}$ C-PBB3 SUVR PET images of AD patients with low (A) and high (B) AS scores. High radioligand retention in the superior sagittal sinus (yellow triangles in panels $a$ and $b$ ) is a non-specific radioactivity accumulation. AD patient with high AS score showed remarkably increased radioligand retention in the vicinity of OFC (yellow arrows in panel b) relative to the patient with low AS score (A). Statistical parametric map illustrates greater accumulations of PBB3-detectable tau lesions in OFC in AD patients with high AS scores relative to those with low AS scores (C). Data are thresholded at false discovery rate-corrected $p$ value $<0.05$ and extent threshold $>600$ voxels $(C) .{ }^{11} \mathrm{C}$ - $P B B 3,{ }^{11} \mathrm{C}$-pyridinyl-butadienyl-benzothiazole 3 ; AD, Alzheimer's disease; AS, Apathy Scale; OFC, orbitofrontal cortex; PET, positron emission tomography; SUVR, standardised uptake value ratio. 
A

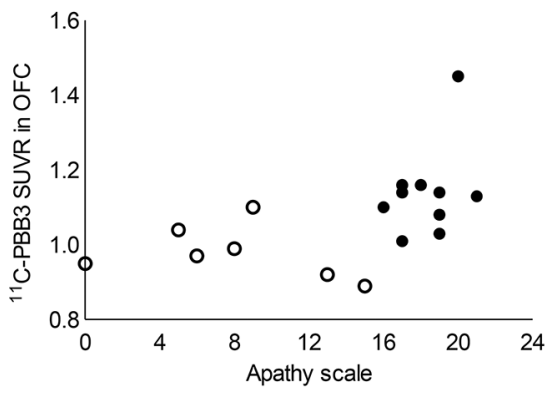

B

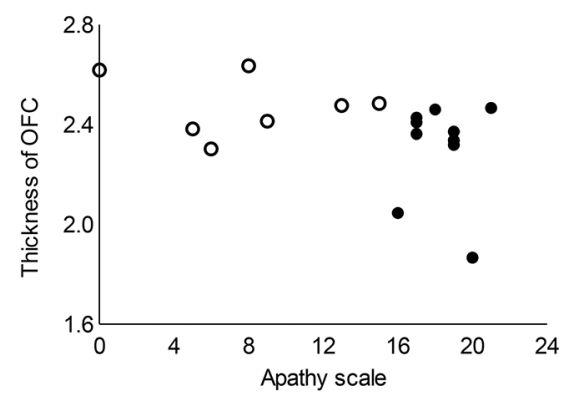

C

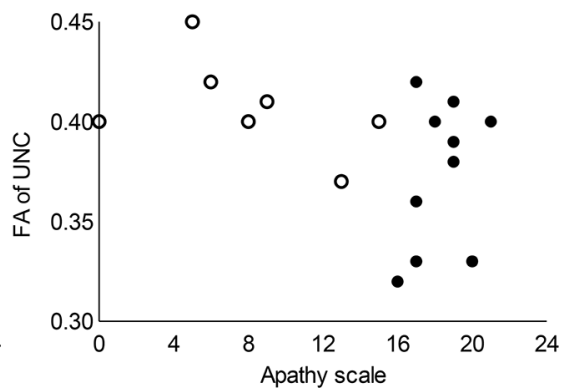

Figure 2 Correlations between AS and neuroimaging measures. (A): mean ${ }^{11} \mathrm{C}-\mathrm{PBB} 3$ SUVR values in OFC showed significant and positive correlation with AS scores $(r=0.53, p=0.037)$. (B and C): mean OFC thickness $(r=-0.53, p=0.033)$ and mean FA value of UNC $(r=-0.51, p=0.044)$ showed significant and negative correlation with AS scores. Mean ${ }^{11} \mathrm{C}$-PiB SUVR values in OFC were not correlated with AS scores ( $p=0.408$; data not shown). Filled and open circles indicate AD patients with high and low AS scores, respectively. ${ }^{11} \mathrm{C}$-PBB3, ${ }^{11} \mathrm{C}$-pyridinyl-butadienyl-benzothiazole $3 ;{ }^{11} \mathrm{C}$-PiB, ${ }^{11} \mathrm{C}$-Pittsburgh compound-B; AS, Apathy Scale; FA, fractional anisotropy; OFC, orbitofrontal cortex; SUVR, standardised uptake value ratio; UNC, uncinate fasciculus.

\section{Path analysis}

Partial correlation analysis with age as covariate demonstrated that mean ${ }^{11} \mathrm{C}-\mathrm{PBB} 3$ SUVR values in OFC were significantly and negatively correlated with mean OFC thickness $(r=-0.73$, $\mathrm{p}=0.001)$ but were not correlated with mean FA values of UNC $(r=-0.33, p=0.209)$. Two path analysis models incorporating these imaging indices could be created to describe apathy (figure 3). Although both path models well described the AS scores (model 1: $\chi^{2}(2)=0.242, p=0.886, C F I=1.000$, RMSEA $<0.001$; model $2: \chi^{2}(2)=0.131, \mathrm{p}=0.718$, CFI $=1.000$, RMSEA <0.001), model 1 (AIC=16.242) showed a better fit than model $2(\mathrm{AIC}=18.131)$. In the first path of model 1 , mean ${ }^{11} \mathrm{C}$-PBB3 SUVR values in OFC were positively correlated with AS scores $(\beta=0.42)$. In the second path of this model, mean ${ }^{11} \mathrm{C}$ PBB3 SUVR values in OFC were negatively correlated with mean thickness of OFC $(\beta=-0.67)$, which further showed a positive correlation with mean FA in UNC $(\beta=0.56)$, and this FA was then negatively correlated with AS scores $(\beta=-0.31)$. We also examined path models without relationships between mean ${ }^{11} \mathrm{C}$ PBB3 SUVR values in OFC and AS scores (data not shown), but these models did not exhibit a better fit than model 1 .
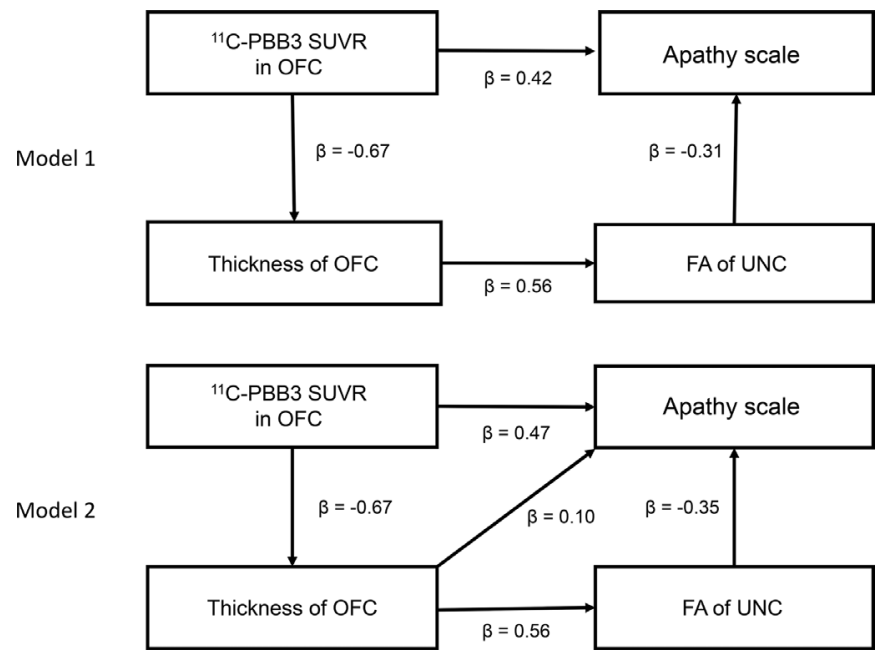

Figure 3 Path analysis models among AS scores and neuroimaging measures. Numbers accompanying arrows are path coefficients. ${ }^{11} \mathrm{C}$-PBB3, ${ }^{11} \mathrm{C}$-pyridinyl-butadienyl-benzothiazole $3 ;{ }^{11} \mathrm{C}$-PiB, ${ }^{11} \mathrm{C}$-Pittsburgh compound-B; AS, Apathy Scale; FA, fractional anisotropy; OFC, orbitofrontal cortex; SUVR, standardised uptake value ratio; UNC, uncinate fasciculus.

\section{DISCUSSION}

The current findings indicate the significance of tau pathologies in OFC detectable by ${ }^{11} \mathrm{C}-\mathrm{PBB} 3-\mathrm{PET}$ as a neuropathological basis of apathy in $\mathrm{AD}$. In addition, the relationship between tau accumulation and apathy is independent of cognitive impairments and depressive state supported by Pearson's correlation analysis, highlighting tau-triggered neurodegenerative processes unique to the pathogenesis of apathy. Neuronal loss in OFC assessed by volumetric MRI and nerve fibre disruptions in UNC quantified by DTI also displayed a tendency to differ between AD cases with high and low AS scores and were significantly correlated with the degree of apathy. These structural alterations were not independent of tau depositions in OFC, since tau PET signals in OFC were intimately associated with OFC thickness, leading to the view that aggregated tau species cause neural death on site. FA values in UNC were closely correlated with OFC thickness rather than tau load in OFC, suggesting that the OFC-UNC connectivity was disrupted by the loss of OFC neurons, which was in turn triggered by tau accumulations.

Correspondingly, a path model supported a mechanistic cascade underlying apathy in $\mathrm{AD}$, which was constituted of the tau pathology in OFC as an initiator, loss of OFC neurons as an intermediate and disconnected OFC-UNC network as a downstream effector on apathy. In this path analysis, model 1 described AS scores with the smallest AIC and consisted of two mechanistic pathways. The first path reflected a direct link of the tau deposition to apathy without structural measures in OFC and UNC. This may imply that PET-detectable tau fibrils give rise to functional deficits in neurons by their neurotoxicity. Apathy has been defined as a decrease of voluntary action and goal-directed behaviour. ${ }^{4}$ OFC is regarded as a region critically involved in decision making, reward processing and emotional control, considered as the association with goal-directed behaviour. ${ }^{35} 36$ Our findings suggested that tau neurotoxicity induces functional deficits in OFC such as synaptic dysfunction, resulting in manifestation of apathy in $\mathrm{AD}$.

The second path in model 1 indicated that the OFC-UNC network abnormality is also critical for the occurrence of apathy. UNC anatomically connects among OFC, temporal cortex and several limbic regions such as the amygdala. ${ }^{33} \mathrm{UNC}$ is a component of Yakovlev's circuit, which is related to language, episodic memory and social emotional processing. ${ }^{10} 37$ Additionally, the amygdala is an important structure for motivation. Previously, structural deteriorations of OFC and UNC were reported to be associated with apathy in frontotemporal degeneration, ${ }^{38}$ which 
is in line with our results. Our findings indicated that tau-induced neuronal death in OFC causes disruption in the associated white matter bundle. This OFC-UNC network abnormality leads to worsened reward and emotional processing, resulting in apathy.

As tau depositions may induce deteriorations of neurons, PBB3 SUVRs could be correlated with general cognitive deficits and disruption of total frontal lobe functions accompanied by depression, and apathetic symptoms might emerge in a manner parallel to the progression of these manifestations. Indeed, the ratio of $\mathrm{AD}$ dementia versus $\mathrm{MCI}$ due to $\mathrm{AD}$ cases differed between the two groups with high and low AS scores, and PBB3-detectable tau burdens in all target ROIs of patients with high AS scores showed a tendency to be greater than those of patients with low AS scores. However, there were no significant differences in the scores of MMSE, FAB and GDS between the two patient groups (table 1). Moreover, these psychometric measures correlated with neither AS scores nor PBB3 SUVR in OFC. These results indicate that apathy at a relatively early clinical stage of $\mathrm{AD}$ is not concurrent with general deteriorations of cognitive and/or frontal lobe-associated functions but may occur in specific relation to tau-provoked OFC deficits.

Additionally, we evaluated path models by incorporating MMSE, GDS and/or FAB scores as model variables. Although we did not find any significant path models including MMSE and/or FAB scores in the current patients with AD, several path models incorporating GDS scores were suggestive of being valid without significance $(\mathrm{p}>0.05)$ of $\chi^{2}$ and RMSEA $<0.05$ (online supplementary figure 1 ). However, AIC values of the path analysis models without GDS (models 1 and 2) were lower than those of the models with GDS. Hence, apathetic symptoms in AD may be better described by local pathological changes in OFC and consequent OFC-UNC disconnections without consideration of the general mood status, while it is yet to be determined whether this notion is adequate throughout the clinical advancement of AD.

As mentioned above, MMSE scores did not correlate with AS scores, unlike a previous work reporting that Apathy Evaluation Scale was associated with MMSE scores in patients with AD. ${ }^{39}$ Although this previous study covered mild, moderate and severe patients with $\mathrm{AD},{ }^{39}$ the subjects included in the present study were mainly patients with early-stage AD spectrum with relatively high MMSE and low GDS scores in a narrow range. Therefore, these distinct clinical stages covered by the two studies may partially explain the somewhat discrepant results. Additionally, the present and previous study evaluated apathetic symptoms by AS and Apathy Evaluation Scale, respectively, and there is a possibility that MMSE scores are differentially associated with these adopted scales.

In contrast with tau burdens, there was no significant difference in PET-visible $A \beta$ accumulations between $A D$ patients with low and high AS scores. This is in agreement with previous reports documenting that $A \beta$ deposits measured by PET reached a plateau at an MCI stage. ${ }^{40}$ Thus, $A \beta$ pathologies at a steadystate level along the transition from MCI to early AD dementia may not overtly influence apathetic statuses. Our result is in line with previous reports. Judging from above-mentioned result, we considered that $\mathrm{A} \beta$ accumulation would not have any influence on the current findings.

Furthermore, we did not find any significant association between AS and ${ }^{11} \mathrm{C}-\mathrm{PiB}$ SUVR values in OFC. This is in agreement with the notion that tau lesions are more clearly implicated in neurodegenerative processes than $\mathrm{A} \beta$ plaques in $\mathrm{AD}$. Indeed, some previous studies reported that the $A \beta$ burden was not associated with cognitive dysfunctions in the course of $\mathrm{AD},{ }^{41}$ and our results may support that there exists a tight link of tau rather than $\mathrm{A} \beta$ pathologies to BPSD including apathy. However, other studies reported that the $A \beta$ burden was related to apathy. ${ }^{42} 43$ This discrepancy may be due to differences in the range of $\mathrm{PiB}$ retention and tau burdens in these studies.

The present study is burdened by the limitation of small sample size. Since we employed relatively conservative methods for statistical tests here, we regarded our positive results as reliable despite the small number of subjects highlighting OFC as an anatomical structure intimately linked to apathetic manifestations. Therefore, we subsequently investigated structural alterations by focusing on the OFC and UNC. Furthermore, even $\mathrm{AD}$ with low $\mathrm{AS}$ scores as well as $\mathrm{AD}$ with high $\mathrm{AS}$ scores showed significantly elevated PBB3 SUVRs in some brain regions compared with cognitively healthy controls (table 2 ), suggesting that the statistical manner used in the present study was not too conservative. Whether focal damage and fibre disruption in other cortical regions affect apathy in $\mathrm{AD}$, however, is indeterminate. Exhaustive structural assays covering extensive brain areas should be performed with larger numbers of subjects and by statistical tests with adequate liberality in future examinations.

In conclusion, our findings highlight the significance of the PET-visible tau tangle formation in OFC and the resultant disruption of the OFC-UNC network as pathogenetic elements of apathy. Similar sequential inductions of tau depositions, local neuronal loss and disconnection of associated nerve fibres could be responsible for other diverse non-cognitive symptoms of $\mathrm{AD}$, and such mechanisms would be identifiable by neuroimaging approaches as employed here. The current data also raise the possibility that emerging antitau drugs would be efficacious for suppressing apathy, if the structural and functional integrities of the OFC-UNC system can be therapeutically rescued. In this context, combined tau PET and MRI assays will offer means to monitor the upstream to downstream processes crucially affecting apathetic symptoms along the course of $\mathrm{AD}$ in both the absence and presence of therapeutic interventions.

\section{Author affiliations}

'Department of Functional Brain Imaging Research, National Institute of Radiologica Sciences, National Institutes for Quantum and Radiological Science and Technology,

Chiba, Japan

${ }^{2}$ Department of Psychiatry, Nara Medical University, Kashihara, Japan

${ }^{3}$ Department of Neurology and Gerontology, Kyoto Prefectural University of Medicine, Kyoto, Japan

${ }^{4}$ Division of Neurology, Kobe University Graduate School of Medicine, Kobe, Japan ${ }^{5}$ Neurology Chiba Clinic, Chiba, Japan

${ }^{6}$ Department of Neurology, Graduate School of Medicine, Chiba University, Chiba, Japan

${ }^{7}$ Department of Clinical and Experimental Neuroimaging, Center for Development of Advanced Medicine for Dementia, National Center for Geriatrics and Gerontology, Obu, Japan

${ }^{8}$ Department of Radiopharmaceutics Development, National Institute of Radiological Sciences (NIRS), National Institutes for Quantum and Radiological Science and Technology (QST), Chiba, Japan

Acknowledgements The authors would like to thank all patients and their caregivers for participation in this study. The authors also acknowledge the support of K. Suzuki, S. Kawakami, J. Ichikawa, and the radiochemistry staffs and radiological technologists at NIRS (no particular order). The authors acknowledge support for the recruitment of patients with cognitive decline of $Y$. Yoshiyama at the Inage Neurology and Memory Clinic, K. Kashiwado at Kashiwado Hospital, and K. Suzuki at the Shirogane Orthopedics Clinic

Contributors Authors' contributions are as follows: (1) substantial contributions to conception and design, or acquisition of data, or analysis and interpretation of data: SKi and HS; (2) drafting the article or revising it critically for important intellectual content: all authors; (3) final approval of the version to be published: all authors 
Funding This work was partly supported by JSPS KAKENHI Grant-in-Aid for Young Scientists (B) (JP15K19756) to SoK from the Japan Society for the Promotion of Science; the Young Scientists (A) (26713031) to H Shimada from the Ministry of Education, Culture, Sports, Science and Technology, Japan; the Kashiwado Memorial Foundation to H Shimada; the Brain Mapping by Integrated Neurotechnologies for Disease Studies (Brain/MINDS) and AMED under Grant Number JP17dm0207007.

Competing interests $\mathrm{H}$ Shimada, M-RZ, TS and MH hold a patent on compounds related to the present report (JP 5422782/EP 12884 742.3).

Patient consent Not required.

Ethics approval This study was approved by the Institutional Review Board of the National Institute of Radiological Sciences (NIRS), in accordance with the ethical code of NIRS and the ethical guidelines for clinical studies presented by the Ministry of Health, Labour and Welfare in Japan, as well as the Declaration of Helsinki. Written informed consent was obtained from all patients and from their spouses or other close family members. The study was registered with UMIN Clinical Trials Registry (UMIN-CTR; number 000009863).

Provenance and peer review Not commissioned; externally peer reviewed.

Open access This is an open access article distributed in accordance with the Creative Commons Attribution Non Commercial (CC BY-NC 4.0) license, which permits others to distribute, remix, adapt, build upon this work non-commercially, and license their derivative works on different terms, provided the original work is properly cited and the use is non-commercial. See: http://creativecommons.org/ licenses/by-nc/4.0/

(c) Article author(s) (or their employer(s) unless otherwise stated in the text of the article) 2018. All rights reserved. No commercial use is permitted unless otherwise expressly granted.

\section{REFERENCES}

1 Fischer CE, Ismail Z, Schweizer TA. Delusions increase functional impairment in Alzheimer's disease. Dement Geriatr Cogn Disord 2012;33:393-9.

2 Ismail Z, Smith EE, Geda Y, et al. Neuropsychiatric symptoms as early manifestations of emergent dementia: Provisional diagnostic criteria for mild behavioral impairment. Alzheimers Dement 2016;12:195-202.

3 Hurt C, Bhattacharyya S, Burns A, et al. Patient and Caregiver Perspectives of Quality of Life in Dementia. Dement Geriatr Cogn Disord 2008:26:138-46.

4 Levy R, Dubois B. Apathy and the functional anatomy of the prefrontal cortex-basal ganglia circuits. Cereb Cortex 2006;16:916-28.

5 Bullock R, Lane R. Executive dyscontrol in dementia, with emphasis on subcortical pathology and the role of butyrylcholinesterase. Curr Alzheimer Res 2007;4:277-93.

6 Toba K, Nakai R, Akishita M, et al. Vitality Index as a useful tool to assess elderly with dementia. Geriatrics and Gerontology International 2002;2:23-9.

7 Holthoff VA, Beuthien-Baumann B, Kalbe E, et al. Regional cerebral metabolism in early Alzheimer's disease with clinically significant apathy or depression. Biol Psychiatry 2005;57:412-21.

8 Lanctôt KL, Moosa S, Herrmann N, et al. A SPECT study of apathy in Alzheimer's disease. Dement Geriatr Cogn Disord 2007:24:65-72.

9 Tunnard C, Whitehead D, Hurt C, et al. Apathy and cortical atrophy in Alzheimer's disease. Int J Geriatr Psychiatry 2011;26:741-8.

10 Hahn C, Lim HK, Won WY, et al. Apathy and white matter integrity in Alzheimer's disease: a whole brain analysis with tract-based spatial statistics. PLoS One 2013:8:e53493-6.

11 Hollocks MJ, Lawrence AJ, Brookes RL, et al. Differential relationships between apathy and depression with white matter microstructural changes and functional outcomes. Brain 2015;138:3803-15.

12 Braak H, Braak E. Staging of Alzheimer's disease-related neurofibrillary changes. Neurobiol Aging 1995;16:271-8

13 Maruyama M, Shimada H, Suhara T, et al. Imaging of tau pathology in a tauopathy mouse model and in Alzheimer patients compared to normal controls. Neuron 2013;79:1094-108.

14 Ono M, Sahara N, Kumata K, et al. Distinct binding of PET ligands PBB3 and AV-145 to tau fibril strains in neurodegenerative tauopathies. Brain 2017;140:aww339-780.

15 Shimada $\mathrm{H}$, Kitamura S, Shinotoh $\mathrm{H}$, et al. Association between $\mathrm{A} \beta$ and tau accumulations and their influence on clinical features in aging and Alzheimer's disease spectrum brains: A [ $\left.{ }^{11} \mathrm{C}\right]$ PBB3-PET study. Alzheimer \& Dementia 2017;2017:11-20.
16 Marin RS. Apathy: a neuropsychiatric syndrome. J Neuropsychiatry Clin Neurosci 1991;3:243-54

17 Starkstein SE, Mayberg HS, Preziosi TJ, et al. Reliability, validity, and clinical correlates of apathy in Parkinson's disease. J Neuropsychiatry Clin Neurosci 1992;4:134-9.

18 McKhann G, Drachman D, Folstein M, et al. Clinical diagnosis of Alzheimer's disease: report of Health and Human Services Task Force on Alzheimer's disease. Neurology 1984;34:939-44

19 Petersen RC, Smith GE, Waring SC, et al. Mild cognitive impairment: clinical characterization and outcome. Arch Neurol 1999;56:303-8.

20 Folstein MF, Folstein SE, McHugh PR. "Mini-mental state". A practical method for grading the cognitive state of patients for the clinician. J Psychiatr Res 1975;12:189-98.

21 Morris JC. The Clinical Dementia Rating (CDR): current version and scoring rules. Neurology 1993;43:2412.

22 Yesavage JA, Brink TL, Rose TL, et al. Development and validation of a geriatric depression screening scale: a preliminary report. J Psychiatr Res 1982;17:37-49.

23 Kobayashi S, Aoki K, Suyama N, et al. Assessment of motivational loss in poststroke patients using the Japanese version of Starkstein's Apathy Scale. Nosotchu 1998:20:318-23.

24 Starr JM, Lonie J. Relationship between behavioural and psychological symptoms of dementia and cognition in Alzheimer's disease. Dement Geriatr Cogn Disord 2007;24:343-7.

25 Hashimoto $\mathrm{H}$, Kawamura $\mathrm{K}$, Takei $\mathrm{M}$, et al. Identification of a major radiometabolite of [11C]PBB3. Nucl Med Biol 2015;42:905-10.

26 Kimura Y, Ichise $M$, Ito $H$, et al. PET Quantification of Tau Pathology in Human Brain with 11C-PBB3. J Nucl Med 2015;56:1359-65.

27 Ashburner J. A fast diffeomorphic image registration algorithm. Neuroimage 2007;38:95-113

28 Tzourio-Mazoyer N, Landeau B, Papathanassiou D, et al. Automated anatomical labeling of activations in SPM using a macroscopic anatomical parcellation of the MN MRI single-subject brain. Neuroimage 2002;15:273-89.

29 Dale AM, Fischl B, Sereno Ml, et al. Segmentation and surface reconstruction. Neuroimage 1999;9:1-16

30 Fischl B, Dale AM. Measuring the thickness of the human cerebral cortex from magnetic resonance images. Proc Nat/ Acad Sci U S A 2000;97:11050-5.

31 Desikan RS, Ségonne F, Fischl B, et al. An automated labeling system for subdividing the human cerebral cortex on MRI scans into gyral based regions of interest. Neuroimage 2006:31:968-80.

32 Smith SM. Fast robust automated brain extraction. Hum Brain Mapp 2002:17:143-55.

33 Taoka T, Morikawa M, Akashi T, et al. Fractional anisotropy--threshold dependence in tract-based diffusion tensor analysis: evaluation of the uncinate fasciculus in Alzheimer disease. AJNR Am J Neuroradiol 2009:30:1700-3.

34 Kiuchi K, Morikawa M, Taoka T, et al. Abnormalities of the uncinate fasciculus and posterior cingulate fasciculus in mild cognitive impairment and early Alzheimer's disease: a diffusion tensor tractography study. Brain Res 2009;1287:184-91.

35 Rahman S, J Sahakia B, N Cardinal R, et al. Decision making and neuropsychiatry. Trends Cogn Sci 2001;5:271-7

36 Strenziok M, Pulaski S, Krueger F, et al. Regional brain atrophy and impaired decision making on the balloon analog risk task in behavioral variant frontotemporal dementia. Cogn Behav Neurol 2011;24:59-67.

37 Von Der Heide RJ, Skipper LM, Klobusicky E, et al. Dissecting the uncinate fasciculus: disorders, controversies and a hypothesis. Brain 2013;136:1692-707.

38 Massimo L, Powers JP, Evans LK, et al. Apathy in Frontotemporal Degeneration: Neuroanatomical Evidence of Impaired Goal-directed Behavior. Front Hum Neurosci 2015;9:611.

39 Stella F, de Andrade LP, Vital TM, et al. Apathy in Alzheimer's disease: Contribution to a clinical view on progression of dementia. Dement Neuropsychol 2010;4:188-93.

40 Jack CR, Knopman DS, Jagust WJ, et al. Tracking pathophysiological processes in Alzheimer's disease: an updated hypothetical model of dynamic biomarkers. Lancet Neurol 2013;12:207-16.

41 Jack CR, Wiste HJ, Weigand SD, et al. Age, Sex, and APOE \&4 Effects on Memory, Brain Structure, and $\beta$-Amyloid Across the Adult Life Span. JAMA Neurol 2015;72:511-9.

42 Marshall GA, Donovan NJ, Lorius $\mathrm{N}$, et al. Apathy is associated with increased amyloid burden in mild cognitive impairment. J Neuropsychiatry Clin Neurosci 2013;25:302-7.

43 Mori T, Shimada $\mathrm{H}$, Shinotoh $\mathrm{H}$, et al. Apathy correlates with prefrontal amyloid $\beta$ deposition in Alzheimer's disease. J Neurol Neurosurg Psychiatry 2014;85:449-55. 